\title{
EVALUATION OF LAND SUITABILITY FOR INCREASING PRODUCTIVITY IN DEGRADED UNDA WATERSHED, DISTRICT OF KARANGASEM, BALI
}

\author{
Ni Made Trigunasih*, I Nyoman Merit, Wiyanti, I Wayan Narka, and \\ I Nyoman Dibia \\ Study Program of Agroecotechnology, Faculty of Agriculture, Udayana University \\ *Corresponding author: trigunasih @unud.ac.id
}

\begin{abstract}
Evaluation of land suitability to increase the productivity of degraded lands in the Unda watershed aims: providing a database of land suitability for land use planning in support of sustainable agricultural development, providing spatial information, and recommendations in accordance with the land use plan of inhibiting factors that exist. Identification of characteristic land quality evaluation survey carried out by the method of land with exploration area of research followed by soil sampling at some sample of land units. Land suitability classification using the criteria of the Technical Guidelines for Evaluation of Land For Agricultural Commodities of Soil Research Institute (2003) is by matching between the haracteristics of the land with the requirements of growing plants was evaluated. Types of agricultural crops that are evaluated include plantation crops and food crops hotikultura fruits. Suitability actual land (land suitability based on survey data) are mostly classified as unsuitable (N), and marginally suitable (S3). Qualities/characteristics as the land that is the main limiting factor, among others: quality of land erosion, slope, water availability, temperature, rooting media (soil texture and coarse material). Sustainable land use planning can be recommended with agroforestry, accompanied by vegetative conservation measures because it is cheaper and easier to be carried out by farmers. Recommended cultivation effort is a combination of timber plants with horticultural crops of fruits with the provisions of at least $40 \%$ must be enclosed timber. Plant timber that can be developed between the other plants that are not too heavy canopy.
\end{abstract}

Keywords: Land suitability, land productivity, land degradation, Unda watershed

\section{INTRODUCTION}

Unda watershed is the largest watershed in Bali Province with 91,585 ha of land dominated by volcanic formation. Land use is $79.59 \%$ including dry land; $68.41 \%$ of the area consist of Regosol soils that are highly susceptible to erosion; and
$45.64 \%$ of its territory are hilly to mountainous, and there are development of illegally $\mathrm{C}$ digging sites in some places. The results of monitoring conducted by Unda Anyar Watershed Management Center in cooperation with Faculty of 
Agriculture Udayana University (2013), criteria and guidelines for controlling and stated that the Unda watershed belongs to conserving agricultural land based on sitethe first priority that must be immediately specific watersheds, as well as technical restored.

guidance, (5) Generate policies, programs

The selection of this research area is and activities that can be offered to local based on the physical condition of the Unda governments related to the control of land watershed as mentioned above. Land damage and Increased productivity of productivity to support optimal production agricultural land, and (6) Provide spatial has been degraded / decreased. . In information in the form of land suitability addition, information on of land resource at maps, erosion hazard maps, and land use the micro-scale level is limited, and still planning maps.

low level of land management / Adequate databases on semidetail / management applied. The reason for choosing food crops and plantations to be evaluated is that most of the watershed area of Unda is dominated by dryland agriculture in mixed and cultivated fields. available on a scale of scale (scale 1: The specific objectives of this $250,000)$ so it is still difficult to apply in the research were (1) Increase the productivity field. Therefore, the provision of a database and quality of degraded agricultural land to on the potential and pattern of land support national food security, (2) Establish management in a larger scale is absolutely formulation of land-based area-specific necessary as a reference in land land-use management practices, (3) management in a watershed, especially in Develope a watershed-based land suitability the Unda watershed which is highly database for urban planning (4) Establish vulnerable to land degradation. 
This land suitability evaluation finding of limiting factors that must be study is very urgent to be done in relation to addressed in sustainable land management; the sustainability of land resources in (3) the formulation of recomendations in supporting sustainable agriculture and food the use of land based on the security at the regional and national levels. potential/suitability of land and constraints The existence of a sustainable agriculture owned by the land concerned. sustainable food protection law (Law No. 41 of 2007) is one of the answers, which needs to be supplemented by various government regulations, ministerial Management Unit (SWP) is astronomically regulations and regional head regulations. located on $08^{\circ} 09^{\prime} 36$ "LS to $08^{\circ} 344^{\prime} 48^{\prime \prime}$ LS Based on the national strategic and $115^{\circ} 23^{\prime} 24$ "BT to $115^{\circ} 42^{\prime} 36^{\prime \prime}$ BT. issues related to recent disasters such as Administratively includes Buleleng District landslide, erosion, flood, drought, volcanic (Tejakula District), Bangli District disaster, etc., a study titled land suitability (Kintamani Sub-district), Klungkung evaluation for increasing degraded land Regency (Dawan and Klungkung Subproductivity is a very urgent study to districts) and Karangasem District undertake. The results of this study are (Bebandem, Rendang, Selat, Manggis, expected to provide solutions on Sidemen, Karangasem, Abang, Kubu Subrehabilitation and conservation of land districts) is 91,585 ha (BP DAS Unda resources, increase productivity with input Anyar, 2010).

in accordance with existing land Land and water resource boundaries.

management planning activities at SWP

The aim of the project are : (1) the DAS Unda are conducted through the compilation of land suitability maps; (2) the approach of land survey unit (the smallest 
unit having the same/similar characteristics the land suitability classification system that can still be limited in accordance with compiled by Puslittanak (1993).

the scale of the map created). Further more, conformity evaluation activities for various food crops and plantation commodities as well as soil and water conservation measures/land management are required to be conducted on each surveyed land unit for the concerned.

The series of activities in this study include: collection and evaluation of secondary data (literature study, traceability of previous documents/reports) as well as primary data (through field survey activities, soil sampling, identification of environmental conditions and current land management, and Conducting interviews with local communities/farmers on issues, and constraints faced, in current resource management.

Evaluation of land suitability is done by matching between the requirements of growing plants with the characteristics/quality of existing land. The land suitability classification criteria used is
Land use plans for each unit of land are prepared based on the evaluation of land suitability, prediction of erosion hazard and the amount of sedimentation occurring. The basic principle of drafting the land use plan used is increasing agricultural production both physically and economically, sustainably, without damaging the environment. In other words land use in accordance with the ability/ suitability of land.

\section{RESULTS AND DISCUSSION}

\section{Characteristics / Land Quality of}

\section{Research Area}

Based on the analysis of soil samples, conducted in Soil and Environmental Science Laboratory Faculty of Agriculture Udayana University, soil 
physical properties such as soil texture is dominated by slightly coarse texture (sandy loam) with sand content $40-70 \%$, medium texture (clay and clay dust) and slightly smooth texture (clay); Crude material ranges from $10-60 \%$; Soil permeability ranges from very fast to moderately fast; Soil erodibility (soil sensitivity to erosion) ranges from moderate to somewhat high; And the erosion hazard rate of $87 \%$ is heavy to very severe.

The chemical properties and soil fertility covering soil CEC (cation exange capasity) varies from 36.92 me 100 g-1 (low) to 84.3 me 100 g-1 (high); base saturation are > $150 \%$ (highly), soil $\mathrm{pH}$ classified are 6.1-7.1 (neutral), salt content are 0.85 mmhos (very low) to 3.49 mmhos (high), C- organic are $5.70 \%$ (low) to 37.21 $\%$ (high), Total-N are $0.50 \%$ (very low) to $0.18 \%$ (low), P-available are $1.51 \mathrm{ppm}$ (very low) to $160.17 \mathrm{ppm}$ (high); And Kavailable are $80.90 \mathrm{ppm}$ (low) to 290.78 ppm (high).
Based on agroclimate data including annual mean temperature, rainfall and dry month taken from several nearby climatological stations, the average annual temperature ranges from $19.5-260 \mathrm{C}$, dry month $(<75 \mathrm{~mm})$ ranges from $0-5$ months, mean rainfall Annual range from $1555 \mathrm{~mm}$ - $3328 \mathrm{~mm}$.

\section{Land Suitability}

The determination of the actual land suitability class/subclass using a matching system is to combine and compare the characteristics/quality of the land with the crop requirements of the evaluated plant commodities. Land suitability criteria use technical land evaluation guidelines for agricultural commodities from Soil Research Institute (2003). In this matching system, the minimum law of land suitability is determined by the smallest value of the land characteristics/qualities as the heaviest or hardest to overcome compared to other limiting factors. 
The type of plant commodity and dry moon), temperature, rooting evaluated is the commodity crop that has medium (soil texture and coarse material). been developed/cultivated on each unit of As previously mentioned, the land concerned. Plantation commodities are research area, especially dry land, is widely developed in research areas sch as: dominated by wavy area form up to coconut, cocoa, clove, and robusta coffee. mountain with slope range of $15 \rightarrow 65 \%$ Fruit horticulture plants include: banana, with erosion hazard level is heavy to very orange, durian, jackfruit, salak, rambutan, heavy. Besides, there is no adequate soil mangosteen and mango. The result of actual conservation measures either mechanically and potential land suitability evaluation or vegetatively.

based on the result of matching the

The quality of land availability of quality/characteristics of the land with some commodities evaluated on each unit of land can be seen in Table 1 .

water (rainfall) is a limiting factor, especially in the upstream region due to high rainfall exceeding that required for horticultural crops of fruits. This proved to

\section{Actual Land Suitability}

be very rare to find the type of fruit plants

Based on the result of matching that developed in the study area. The between the quality/characteristics of the land and the evaluated plant commodities, actual land suitability is largely classified as unsuitable $(\mathrm{N})$, and marginally appropriate (S3). The quality/characteristics of the land that are the main obstacle factors are: the quality of water availability (duration of dry months) is a limiting factor due to the long dry months (5 months) for the lower research area). For forest timber species the quality of land availability is not too problematic.

quality of land erosion hazard (slope and erosion hazard), water availability (rainfall 
Table 1. Actual and Potential Land Suitability Evaluation of Several Types of Plantation Crops, Fruits and Forest Crops On Dryland Unda Watershed

\begin{tabular}{|c|c|c|c|c|c|c|c|c|c|}
\hline \multirow{3}{*}{\begin{tabular}{|l} 
No. \\
Sam \\
ple
\end{tabular}} & \multirow{3}{*}{\begin{tabular}{|c|} 
Location \\
District/Village
\end{tabular}} & \multicolumn{8}{|c|}{ Land Suitability for Plantation Crops } \\
\hline & & \multicolumn{4}{|c|}{ Coconut } & \multicolumn{4}{|c|}{ Cacao } \\
\hline & & $\begin{array}{l}\text { A } \\
\text { ct. }\end{array}$ & $\begin{array}{l}\text { Limiting } \\
\text { factor }\end{array}$ & Ptt. & $\begin{array}{l}\text { Limi- } \\
\text { ting } \\
\text { factor }\end{array}$ & Act. & $\begin{array}{l}\text { Limiting } \\
\text { factor }\end{array}$ & Ptt. & $\begin{array}{l}\text { Limiting } \\
\text { factor }\end{array}$ \\
\hline 1 & Kintamani/Suter & $\mathrm{N}$ & $\mathrm{t}$ & $\mathrm{N}$ & $\mathrm{t}$ & $\mathrm{N}$ & $\mathrm{t}, \mathrm{lp} 1$ & $\mathrm{~N}$ & $\mathrm{t}$ \\
\hline 2 & Rendang/Pempatan & S3 & $\mathrm{t}$ & S3 & $\mathrm{t}$ & S3 & $\mathrm{w} 1, \mathrm{r} 1$ & $\mathrm{~S} 2$ & $\mathrm{w} 1, \mathrm{r} 1$ \\
\hline 3 & Rendang/Besakih & S3 & $\mathrm{e} 1.2$ & S3 & $\mathrm{t}$ & S3 & $\mathrm{e} 1.2, \mathrm{r} 2$ & S3 & $\mathrm{t}, \mathrm{w} 1$ \\
\hline 4 & Rendang/Menanga & S3 & $\mathrm{t}, \mathrm{e} 2$ & S3 & $\mathrm{t}$ & S3 & $\begin{array}{ll}\text { w1, } & \text { r1, } \\
\text { e2 }\end{array}$ & $\mathrm{S} 2$ & $\mathrm{t}, \mathrm{w} 1 \mathrm{r} 1$ \\
\hline 5 & Selat/Muncan & S3 & e2 & $\mathrm{S} 2$ & $\mathrm{t}, \mathrm{r} 2$ & S3 & $\mathrm{w} 1, \mathrm{r} 1, \mathrm{e} 2$ & $\mathrm{~S} 2$ & $\mathrm{t}, \mathrm{w} 1, \mathrm{r} 1$ \\
\hline 6 & Selat/Mertabuana & S3 & e2 & $\mathrm{S} 2$ & $\mathrm{t}$ & S3 & w1, e2 & $\mathrm{S} 2$ & $\mathrm{t}, \mathrm{w} 1$ \\
\hline 7 & Selat/Sebudi & S3 & e2 & S3 & $\mathrm{t}$ & S3 & e2 & $\mathrm{S} 2$ & $\mathrm{t}, \mathrm{w} 1$ \\
\hline 8 & $\begin{array}{l}\text { Sidemen/Sangkan } \\
\text { Gunung }\end{array}$ & $\mathrm{N}$ & $\mathrm{e} 1.2$ & $\mathrm{~N}$ & $\mathrm{e} 1.2$ & $\mathrm{~N}$ & $\mathrm{e} 1.2$ & $\mathrm{~N}$ & $\mathrm{e} 1.2$ \\
\hline 9 & Sidemen/Tangkup & S3 & $\mathrm{e} 1.2$ & $\mathrm{~S} 2$ & $\mathrm{t}, \mathrm{w} 1$ & S3 & $\mathrm{e} 1.2$ & $\mathrm{~S} 2$ & $\mathrm{t}, \mathrm{e} 1$ \\
\hline 10 & Sidemen/Talibeng & S3 & $\mathrm{e} 1$ & $\mathrm{~S} 2$ & $\mathrm{t}, \mathrm{w} 1$ & S3 & $\mathrm{e} 1.2$ & $\mathrm{~S} 2$ & $\mathrm{t}, \mathrm{e} 1$ \\
\hline 11 & Sidemen/Dulah & $\mathrm{N}$ & $\mathrm{e} 1.2$ & $\mathrm{~N}$ & $\mathrm{e} 1.2$ & $\mathrm{~N}$ & w2, e1.2 & $\mathrm{N}$ & $\mathrm{e} 1.2$ \\
\hline 12 & $\begin{array}{l}\text { Sidemen/Telaga } \\
\text { Tawang }\end{array}$ & $\mathrm{N}$ & $\mathrm{e} 1.2$ & $\mathrm{~N}$ & $\mathrm{e} 1.2$ & $\mathrm{~N}$ & w2, e1.2 & $\mathrm{N}$ & e1.2 \\
\hline 13 & Sidemen/Kikian & $\mathrm{N}$ & $\mathrm{e} 1.2$ & $\mathrm{~N}$ & $\mathrm{e} 1.2$ & $\mathrm{~N}$ & w2, e1.2 & $\mathrm{N}$ & $\mathrm{e} 1.2$ \\
\hline 14 & Bebandem/Jungutan & $\mathrm{N}$ & $\mathrm{e} 1.2$ & $\mathrm{~N}$ & e1.2 & $\mathrm{N}$ & w2,e1.2 & $\mathrm{N}$ & $\mathrm{e} 1.2$ \\
\hline 15 & Dawan/Sulang & S3 & $\mathrm{e} 2$ & S2 & w2 & S3 & w2 & S3 & w2 \\
\hline
\end{tabular}

Table 1. (Continued)

\begin{tabular}{|c|c|c|c|c|c|c|c|c|c|}
\hline \multirow{3}{*}{$\begin{array}{l}\text { No. } \\
\text { Sam } \\
\text { ple }\end{array}$} & \multirow{3}{*}{$\frac{\text { Location }}{\text { District/Village }}$} & \multicolumn{8}{|c|}{ Land Suitability for Plantation Crops and Fruit Horticulture } \\
\hline & & \multicolumn{4}{|c|}{ clove } & \multicolumn{4}{|c|}{ Robusta Coffe } \\
\hline & & Act & $\begin{array}{l}\text { Limiting } \\
\text { factor }\end{array}$ & Ptt. & $\begin{array}{l}\text { Limiti } \\
\mathrm{ng} \\
\text { factor }\end{array}$ & Act. & $\begin{array}{l}\text { Limiting } \\
\text { factor }\end{array}$ & Ptt. & $\begin{array}{l}\text { Limiting } \\
\text { factor }\end{array}$ \\
\hline 1 & Kintamani/Suter & $\mathrm{N}$ & $\mathrm{t}$ & $\mathrm{N}$ & $\mathrm{t}$ & $\mathrm{N}$ & $\mathrm{T}$ & S3 & $\mathrm{t}, \mathrm{r} 1, \mathrm{e} 1$ \\
\hline 2 & Rendang/Pempatan & S3 & $\mathrm{w} 1, \mathrm{r} 1$ & S3 & $\begin{array}{l}\mathrm{t}, \mathrm{w} 1, \\
\mathrm{r} 1\end{array}$ & S3 & $\mathrm{r} 1.2$ & $\mathrm{~S} 2$ & $\mathrm{w} 1, \mathrm{r} 1.2$ \\
\hline 3 & Rendang/Besakih & $\mathrm{N}$ & $\mathrm{e} 1.2, \mathrm{r} 2$ & S3 & $\mathrm{t}, \mathrm{w} 1$ & $\mathrm{~N}$ & $\mathrm{e} 1.2$ & S3 & w1 \\
\hline 4 & Rendang/Menanga & S3 & $\mathrm{w} 1, \mathrm{r} 1, \mathrm{e} 2$ & $\mathrm{~S} 2$ & $\begin{array}{l}\mathrm{t}, \mathrm{w} 1, \\
\mathrm{r} 1\end{array}$ & S3 & $\mathrm{r} 1, \mathrm{e} 2$ & $\mathrm{~S} 2$ & $\mathrm{w} 1, \mathrm{r} 1$ \\
\hline 5 & Selat/Muncan & S3 & w1, r1,e2 & $\mathrm{S} 2$ & $\begin{array}{l}\mathrm{t}, \mathrm{w} 1, \\
\mathrm{r} 1\end{array}$ & S3 & $\mathrm{r} 1, \mathrm{e} 2$ & $\mathrm{~S} 2$ & w1,r1 \\
\hline 6 & Selat/Mertabuana & S3 & $\mathrm{w} 1, \mathrm{e} 2$ & $\mathrm{~S} 2$ & $\mathrm{t}, \mathrm{w} 1$ & S3 & $\mathrm{e} 2$ & $\mathrm{~S} 2$ & $\mathrm{w} 1$ \\
\hline 7 & Selat/Sebudi & $\mathrm{N}$ & $\mathrm{e} 2$ & $\mathrm{~S} 2$ & $\mathrm{t}, \mathrm{w} 1$ & $\mathrm{~N}$ & $\mathrm{e} 2$ & $\mathrm{~S} 2$ & $\mathrm{t}, \mathrm{w} 1$ \\
\hline 8 & $\begin{array}{l}\text { Sidemen/Sangkan } \\
\text { Gunung }\end{array}$ & $\mathrm{N}$ & $\mathrm{e} 1.2$ & $\mathrm{~N}$ & $\mathrm{e} 1.2$ & $\mathrm{~N}$ & e. 2 & $\mathrm{~S} 2$ & $\mathrm{r} 2, \mathrm{e} 2$ \\
\hline
\end{tabular}


EVALUATION OF LAND SUITABILITY FOR INCREASING PRODUCTIVITY IN DEGRADED UNDA

WATERSHED, DISTRICT OF KARANGASEM, BALI

Ni Made Trigunasih*, I Nyoman Merit, Wiyanti, I Wayan Narka, and I Nyoman Dibia

\begin{tabular}{|c|c|c|c|c|c|c|c|c|c|}
\hline 9 & Sidemen/Tangkup & S3 & $\mathrm{e} 1.2$ & S2 & $\mathrm{t}, \mathrm{e} 1$ & S3 & $\mathrm{e} 1.2$ & $\mathrm{~S} 2$ & $\mathrm{e} 1$ \\
\hline 10 & Sidemen/Talibeng & S3 & $\mathrm{w} 2, \mathrm{e} 1$ & S2 & $\mathrm{t}, \mathrm{e} 1$ & S3 & $\mathrm{e} 1.2$ & $\mathrm{~S} 2$ & e1 \\
\hline 11 & Sidemen/Dulah & $\mathrm{N}$ & $\mathrm{w} 2, \mathrm{e} 1.2$ & $\mathrm{~N}$ & e1.2 & $\mathrm{N}$ & e2 & S3 & $\mathrm{w} 2, \mathrm{e} 1.2$ \\
\hline 12 & $\begin{array}{l}\text { Sidemen/Telaga } \\
\text { Tawang }\end{array}$ & $\mathrm{N}$ & $\mathrm{w} 2, \mathrm{e} 1.2$ & $\mathrm{~N}$ & e1.2 & $\mathrm{N}$ & $\mathrm{e} 1.2$ & $\mathrm{~N}$ & $\mathrm{e} 1.2$ \\
\hline 13 & Sidemen/Kikian & $\mathrm{N}$ & $\mathrm{w} 2, \mathrm{e} 1.2$ & $\mathrm{~N}$ & e1.2 & $\mathrm{N}$ & e2 & S3 & $\begin{array}{l}\text { w2, e1.2, } \\
\text { r1 }\end{array}$ \\
\hline 14 & $\begin{array}{l}\text { Bebandem/Junguta } \\
\mathrm{n}\end{array}$ & $\mathrm{N}$ & $\mathrm{w} 2, \mathrm{e} 1.2$ & $\mathrm{~N}$ & e1.2 & $\mathrm{N}$ & $\mathrm{e} 2$ & S3 & w2, e1.2 \\
\hline 15 & Dawan/Sulang & $\mathrm{N}$ & w2 & S3 & w2 & S3 & e2 & $\mathrm{S} 2$ & w2 \\
\hline
\end{tabular}

Table 1. (Continued)

\begin{tabular}{|c|c|c|c|c|c|c|c|c|c|}
\hline \multirow{3}{*}{$\begin{array}{l}\text { No. } \\
\text { Sam } \\
\text { ple }\end{array}$} & \multirow{3}{*}{$\frac{\text { Location }}{\text { District/Village }}$} & \multicolumn{8}{|c|}{ Land Suitability for Fruit Horticulture Plants } \\
\hline & & \multicolumn{4}{|c|}{ Banana } & \multicolumn{4}{|c|}{ Orange } \\
\hline & & Act. & $\begin{array}{l}\text { Limiting } \\
\text { Factor }\end{array}$ & Ptt. & $\begin{array}{l}\text { Limitin } \\
\text { g Factor }\end{array}$ & Act. & $\begin{array}{l}\text { Limi- } \\
\text { ting } \\
\text { Factor }\end{array}$ & Ptt. & $\begin{array}{l}\text { Limi- } \\
\text { ting } \\
\text { Factor }\end{array}$ \\
\hline 1 & Kintamani/Suter & S3 & $\begin{array}{ll}\mathrm{t}, & \mathrm{w} 1, \mathrm{r} 1, \\
\mathrm{e} 1.2 & \end{array}$ & S3 & $\mathrm{t}, \mathrm{w} 1, \mathrm{r} 1$ & S3 & e2 & $\mathrm{S} 2$ & $\mathrm{w} 1.2$ \\
\hline 2 & $\begin{array}{l}\text { Rendang/Pempat } \\
\text { an }\end{array}$ & S3 & $\mathrm{r} 1$ & $\mathrm{~S} 2$ & $\mathrm{w} 1, \mathrm{r} 1$ & $\mathrm{~S} 2$ & w1.2 & $\mathrm{S} 2$ & $\mathrm{w} 1.2$ \\
\hline 3 & $\begin{array}{l}\text { Rendang/Besaki } \\
\mathrm{h}\end{array}$ & $\mathrm{N}$ & $\mathrm{r} 2, \mathrm{e} 1.2$ & S3 & $\mathrm{e} 1.2$ & $\mathrm{~N}$ & $\mathrm{e} 1.2$ & S3 & $\mathrm{e} 1.2$ \\
\hline 4 & $\begin{array}{l}\text { Rendang/Menan } \\
\text { ga }\end{array}$ & S3 & w1,r1, e2 & S3 & $\mathrm{w} 1, \mathrm{r} 1$ & S3 & e2 & $\mathrm{S} 2$ & $\mathrm{w} 1.2$ \\
\hline 5 & Selat/Muncan & S3 & $\mathrm{w} 1, \mathrm{r} 1, \mathrm{e} 2$ & S3 & $\mathrm{w} 1, \mathrm{r} 1$ & S3 & e2 & $\mathrm{S} 2$ & $\mathrm{w} 1.2$ \\
\hline 6 & $\begin{array}{l}\text { Selat/Mertabuan } \\
\mathrm{a}\end{array}$ & S3 & w1, e2 & S3 & w1 & S3 & e2 & $\mathrm{S} 2$ & $\mathrm{w} 1.2$ \\
\hline 7 & Selat/Sebudi & $\mathrm{N}$ & e2 & S3 & w1, e2 & $\mathrm{N}$ & $\mathrm{e} 2$ & $\mathrm{~S} 2$ & $\mathrm{w} 1.2$ \\
\hline 8 & $\begin{array}{l}\text { Sidemen/Sangka } \\
\text { n Gunung }\end{array}$ & $\mathrm{N}$ & $\mathrm{e} 1.2$ & $\mathrm{~N}$ & $\mathrm{e} 1.2$ & $\mathrm{~N}$ & $\mathrm{e} 1.2$ & $\mathrm{~N}$ & $\mathrm{e} 1.2$ \\
\hline 9 & $\begin{array}{l}\text { Sidemen/Tangku } \\
\mathrm{p}\end{array}$ & $\mathrm{N}$ & $\mathrm{e} 1.2$ & $\mathrm{~S} 3$ & w1.2 & $\mathrm{N}$ & e2 & $\mathrm{S} 2$ & $\mathrm{e} 1.2$ \\
\hline 10 & $\begin{array}{l}\text { Sidemen/Taliben } \\
\mathrm{g}\end{array}$ & S3 & w2, e1.2 & S3 & w1, e1 & $\mathrm{N}$ & $\mathrm{e} 1.2$ & $\mathrm{~S} 3$ & $\mathrm{e} 1.2$ \\
\hline 11 & Sidemen/Dulah & $\mathrm{N}$ & $\mathrm{e} 1.2$ & $\mathrm{~N}$ & $\mathrm{e} 1.2$ & $\mathrm{~N}$ & $\mathrm{e} 1.2$ & $\mathrm{~N}$ & $\mathrm{e} 1.2$ \\
\hline 12 & $\begin{array}{l}\text { Sidemen/Telaga } \\
\text { Tawang }\end{array}$ & $\mathrm{N}$ & $\mathrm{e} 1.2$ & $\mathrm{~N}$ & $\mathrm{e} 1.2$ & $\mathrm{~N}$ & $\mathrm{e} 1.2$ & $\mathrm{~N}$ & $\mathrm{e} 1.2$ \\
\hline 13 & Sidemen/Kikian & $\mathrm{N}$ & $\mathrm{e} 1.2$ & S3 & e1.2 & $\mathrm{N}$ & $\mathrm{e} 1.2$ & S3 & $\mathrm{e} 1.2$ \\
\hline 14 & $\begin{array}{l}\text { Bebandem/Jungu } \\
\text { tan }\end{array}$ & $\mathrm{N}$ & $\mathrm{e} 1.2$ & $\mathrm{~N}$ & $\mathrm{e} 1.2$ & $\mathrm{~N}$ & $\mathrm{e} 1.2$ & $\mathrm{~N}$ & $\mathrm{e} 1.2$ \\
\hline 15 & Dawan/Sulang & S3 & $\mathrm{w} 2, \mathrm{e} 2$ & S3 & w2 & S3 & $\mathrm{e} 2$ & $\mathrm{~S} 1$ & - \\
\hline
\end{tabular}


Table 1. (Continued)

\begin{tabular}{|c|c|c|c|c|c|c|c|c|c|}
\hline \multirow{3}{*}{$\begin{array}{l}\text { No. } \\
\text { Sam } \\
\text { ple }\end{array}$} & \multirow{3}{*}{\begin{tabular}{|c|} 
Location \\
District/Village
\end{tabular}} & \multicolumn{8}{|c|}{ Land Suitability for Fruit Horticulture Plants } \\
\hline & & \multicolumn{4}{|c|}{ Durian } & \multicolumn{4}{|c|}{ jackfruit } \\
\hline & & Act & $\begin{array}{l}\text { Limitin } \\
\mathrm{g} \\
\text { factor }\end{array}$ & Ptt. & $\begin{array}{l}\text { Limiting } \\
\text { factor }\end{array}$ & Act & $\begin{array}{l}\text { Limiting } \\
\text { factor }\end{array}$ & Ptt. & $\begin{array}{l}\text { Limiting } \\
\text { factor }\end{array}$ \\
\hline 1 & Kintamani/Suter & S3 & $\mathrm{t}, \mathrm{r} 1$ & S3 & $\mathrm{t}$ & S3 & $\mathrm{w} 1, \mathrm{r} 1, \mathrm{e} 2$ & S3 & $\mathrm{w} 1, \mathrm{r} 1$ \\
\hline 2 & $\begin{array}{l}\text { Rendang/Pempata } \\
\mathrm{n}\end{array}$ & S3 & $\mathrm{r} 1.2$ & $\mathrm{~S} 2$ & $\mathrm{t}, \mathrm{r} 1, \mathrm{w} 1$ & S3 & $\mathrm{w} 1, \mathrm{r} 1.2$ & S3 & $\mathrm{w} 1, \mathrm{r} 1$ \\
\hline 3 & Rendang/Besakih & $\mathrm{N}$ & $\mathrm{r} 2, \mathrm{e} 1.2$ & S3 & $\mathrm{t}, \mathrm{e} 12$ & S3 & $\mathrm{r} 2, \mathrm{e} 1.2$ & S3 & $\mathrm{w} 1, \mathrm{e} 1.2$ \\
\hline 4 & $\begin{array}{l}\text { Rendang/Menang } \\
\text { a }\end{array}$ & S3 & $\mathrm{r} 1, \mathrm{e} 2$ & $\mathrm{~S} 2$ & $\mathrm{t}, \mathrm{w} 1, \mathrm{r} 1$ & S3 & r1, w1,e2 & $\mathrm{S} 2$ & $\mathrm{w} 1, \mathrm{r} 1$ \\
\hline 5 & Selat/Muncan & S3 & $\mathrm{r} 1, \mathrm{e} 2$ & $\mathrm{~S} 2$ & $\mathrm{t}, \mathrm{w} 1, \mathrm{r} 1$ & S3 & r1, w1,e2 & $\mathrm{S} 2$ & $\mathrm{w} 1, \mathrm{r} 1$ \\
\hline 6 & Selat/Mertabuana & S3 & e2 & $\mathrm{S} 2$ & $\mathrm{t}, \mathrm{w} 1$ & S3 & $\mathrm{w} 1, \mathrm{e} 2$ & $\mathrm{~S} 2$ & w1 \\
\hline 7 & Selat/Sebudi & $\mathrm{N}$ & e2 & $\mathrm{S} 2$ & $\mathrm{t}, \mathrm{w} 1, \mathrm{e} 2$ & $\mathrm{~N}$ & e2 & $\mathrm{S} 2$ & w1 \\
\hline 8 & $\begin{array}{l}\text { Sidemen/Sangkan } \\
\text { Gunung }\end{array}$ & $\mathrm{N}$ & $\mathrm{e} 1.2$ & $\mathrm{~N}$ & $\mathrm{e} 1.2$ & $\mathrm{~N}$ & $\mathrm{e} 1.2$ & $\mathrm{~N}$ & $\mathrm{e} 1.2$ \\
\hline 9 & Sidemen/Tangkup & $\mathrm{N}$ & $\mathrm{e} 2$ & $\mathrm{~S} 2$ & w1,e1 & $\mathrm{N}$ & $\mathrm{e} 2$ & $\mathrm{~S} 2$ & $\mathrm{e} 1.2$ \\
\hline 10 & Sidemen/Talibeng & $\mathrm{N}$ & $\mathrm{e} 1.2$ & $\mathrm{~N}$ & $\mathrm{e} 1.2$ & $\mathrm{~N}$ & $\mathrm{e} 1.2$ & $\mathrm{~N}$ & e1.2 \\
\hline 11 & Sidemen/Dulah & $\mathrm{N}$ & $\mathrm{e} 1.2$ & $\mathrm{~N}$ & $\mathrm{e} 1.2$ & $\mathrm{~N}$ & $\mathrm{e} 1.2$ & $\mathrm{~N}$ & $\mathrm{e} 1.2$ \\
\hline 12 & $\begin{array}{l}\text { Sidemen/Telaga } \\
\text { Tawang }\end{array}$ & $\mathrm{N}$ & $\mathrm{e} 1.2$ & $\mathrm{~N}$ & $\mathrm{e} 1.2$ & $\mathrm{~N}$ & $\mathrm{e} 1.2$ & $\mathrm{~N}$ & $\mathrm{e} 1.2$ \\
\hline 13 & Sidemen/Kikian & $\mathrm{N}$ & $\mathrm{e} 1.2$ & $\mathrm{~N}$ & $\mathrm{e} 1.2$ & $\mathrm{~N}$ & $\mathrm{e} 1.2$ & $\mathrm{~N}$ & $\mathrm{e} 1.2$ \\
\hline 14 & $\begin{array}{l}\text { Bebandem/Jungut } \\
\text { an }\end{array}$ & $\mathrm{N}$ & $\mathrm{e} 1.2$ & $\mathrm{~N}$ & $\mathrm{e} 1.2$ & $\mathrm{~N}$ & $\mathrm{e} 1.2$ & $\mathrm{~N}$ & $\mathrm{e} 1.2$ \\
\hline 15 & Dawan/Sulang & S3 & w1, e2 & $\mathrm{S} 2$ & w1 & $\mathrm{S} 1$ & - & S1 & - \\
\hline
\end{tabular}

Table 1. (Continued)

\begin{tabular}{|c|c|c|c|c|c|c|c|c|c|}
\hline \multirow{3}{*}{$\begin{array}{l}\text { No. } \\
\text { Sam } \\
\text { pel }\end{array}$} & Location & \multicolumn{8}{|c|}{ Land Suitability for Fruit Horticulture Plants } \\
\hline & \multirow[t]{2}{*}{ District/Village } & \multicolumn{4}{|c|}{ Salak } & \multicolumn{4}{|c|}{ Rambutan } \\
\hline & & Act & $\begin{array}{l}\text { Limiting } \\
\text { factor }\end{array}$ & Ptt. & $\begin{array}{l}\text { Limitin } \\
\mathrm{g} \\
\text { factor }\end{array}$ & Act & $\begin{array}{l}\text { Limitin } \\
\mathrm{g} \\
\text { factor }\end{array}$ & Ptt. & $\begin{array}{l}\text { Limitin } \\
\mathrm{g} \\
\text { factor }\end{array}$ \\
\hline 1 & Kintamani/Suter & S3 & $\begin{array}{l}\text { w1,r1, } \\
\text { e1.2 }\end{array}$ & S3 & w1 & S3 & $\begin{array}{lr}\mathrm{t}, & \mathrm{r} 1, \\
\mathrm{e} 1.2 & \end{array}$ & S3 & t. \\
\hline 2 & $\begin{array}{l}\text { Rendang/Pempata } \\
\mathrm{n}\end{array}$ & $\mathrm{N}$ & r2 & S3 & w1, r1.2 & S3 & $\mathrm{r} 1$ & $\mathrm{~S} 2$ & w1, r1 \\
\hline 3 & Rendang/Besakih & $\mathrm{N}$ & $\mathrm{r} 2, \mathrm{e} 1.2$ & S3 & $\mathrm{w} 1, \mathrm{e} 1.2$ & $\mathrm{~N}$ & $\mathrm{e} 1.2$ & $\mathrm{~S} 2$ & w1,e1.2 \\
\hline 4 & Rendang/Menanga & S3 & $\mathrm{w} 1, \mathrm{r} 1, \mathrm{e} 2$ & S3 & $\mathrm{w} 1, \mathrm{r} 1$ & S3 & $\mathrm{e} 2$ & $\mathrm{~S} 2$ & w1.r1 \\
\hline 5 & Selat/Muncan & S3 & $\mathrm{w} 1, \mathrm{r} 1, \mathrm{e} 2$ & S3 & $\mathrm{w} 1, \mathrm{r} 1$ & S3 & $\mathrm{e} 2$ & $\mathrm{~S} 2$ & w1.r1 \\
\hline 6 & Selat/Mertabuana & S3 & $\mathrm{w} 1, \mathrm{e} 2$ & S3 & w1 & S3 & $\mathrm{e} 2$ & $\mathrm{~S} 2$ & w1 \\
\hline 7 & Selat/Sebudi & $\mathrm{N}$ & e2 & S3 & w1, e2 & $\mathrm{N}$ & $\mathrm{e} 2$ & $\mathrm{~S} 2$ & w1 \\
\hline
\end{tabular}




\begin{tabular}{|l|l|l|l|l|l|l|l|l|l|}
\hline 8 & $\begin{array}{l}\text { Sidemen/Sangkan } \\
\text { Gunung }\end{array}$ & $\mathrm{N}$ & $\mathrm{e} 1.2$ & $\mathrm{~N}$ & $\mathrm{e} 1.2$ & $\mathrm{~N}$ & $\mathrm{e} 1.2$ & $\mathrm{~N}$ & $\mathrm{e} 1.2$ \\
\hline 9 & Sidemen/Tangkup & $\mathrm{N}$ & $\mathrm{e} 2$ & $\mathrm{~S} 2$ & $\mathrm{e} 1.2$ & $\mathrm{~N}$ & $\mathrm{e} 1.2$ & $\mathrm{~S} 2$ & $\mathrm{e} 1.2$ \\
\hline 10 & Sidemen/Talibeng & $\mathrm{N}$ & $\mathrm{e} 1$ & $\mathrm{~N}$ & $\mathrm{e} 1$ & $\mathrm{~N}$ & $\mathrm{e} 1.2$ & $\mathrm{~N}$ & $\mathrm{e} 1.2$ \\
\hline 11 & Sidemen/Dulah & $\mathrm{N}$ & $\mathrm{e} 1.2$ & $\mathrm{~N}$ & $\mathrm{e} 1.2$ & $\mathrm{~N}$ & $\mathrm{e} 1.2$ & $\mathrm{~N}$ & $\mathrm{e} 1.2$ \\
\hline 12 & $\begin{array}{l}\text { Sidemen/Telaga } \\
\text { Tawang }\end{array}$ & $\mathrm{N}$ & $\mathrm{e} 1.2$ & $\mathrm{~N}$ & $\mathrm{e} 1.2$ & $\mathrm{~N}$ & $\mathrm{e} 1.2$ & $\mathrm{~N}$ & $\mathrm{e} 1.2$ \\
\hline 13 & Sidemen/Kikian & $\mathrm{N}$ & $\mathrm{e} 1.2$ & $\mathrm{~S} 3$ & $\mathrm{e} 1.2$ & $\mathrm{~N}$ & $\mathrm{e} 1.2$ & $\mathrm{~S} 3$ & $\mathrm{e} 1.2$ \\
\hline 14 & $\begin{array}{l}\text { Bebandem/Junguta } \\
\mathrm{n}\end{array}$ & $\mathrm{N}$ & $\mathrm{e} 1.2$ & $\mathrm{~N}$ & $\mathrm{e} 1.2$ & $\mathrm{~N}$ & $\mathrm{e} 1.2$ & $\mathrm{~N}$ & $\mathrm{e} 1.2$ \\
\hline 15 & Dawan/Klungkung & $\mathrm{S} 3$ & $\mathrm{e} 2$ & $\mathrm{~S} 1$ & - & $\mathrm{S} 3$ & w1, e2 & S2 & $\mathrm{w} 1$ \\
\hline
\end{tabular}

Table 1. (Continued)

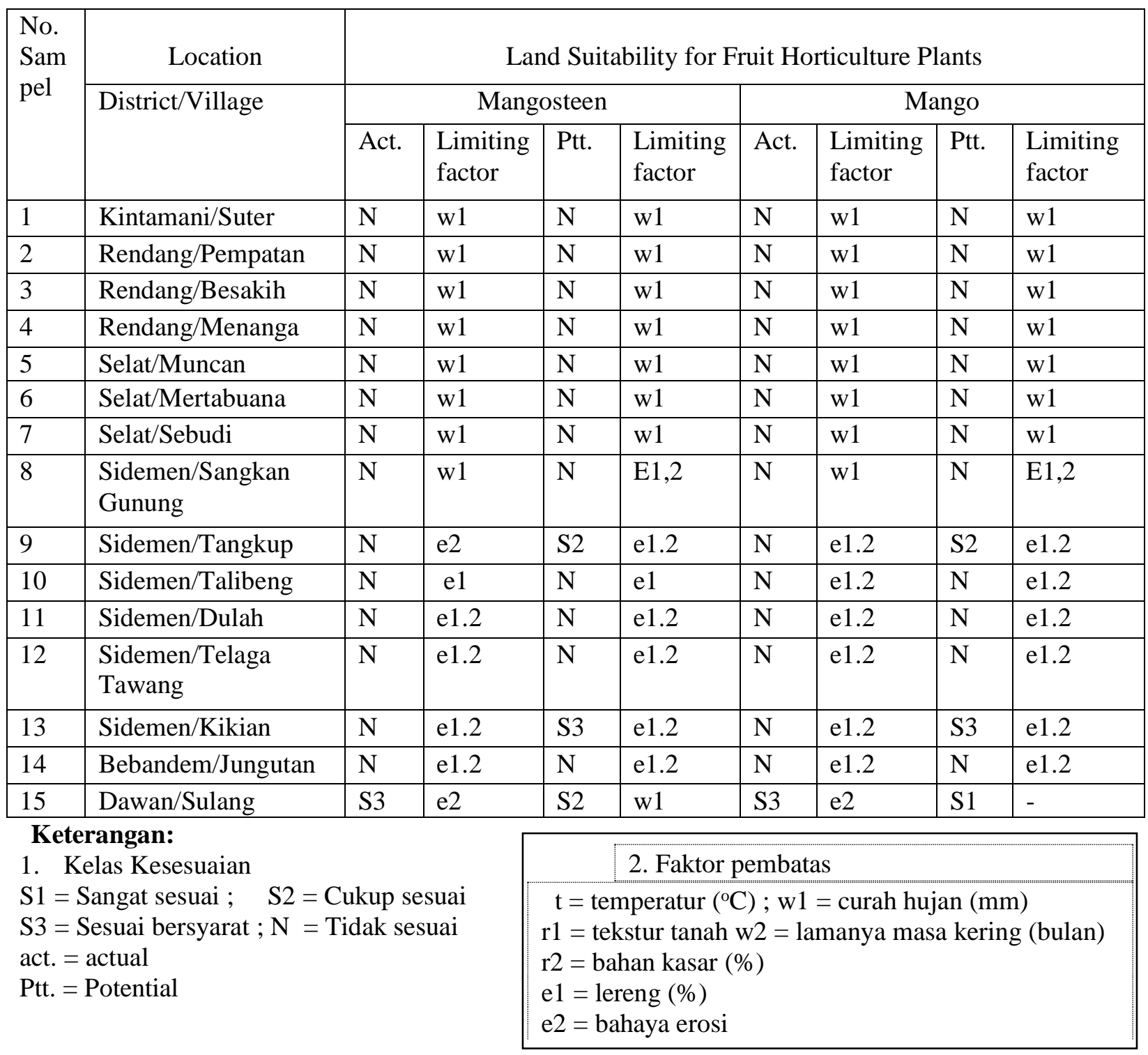




\section{Potential Land Suitability}

Based on the assumption of the type of improvement effort that can be done on the quality/characteristics of the land that is an inhibiting factor, potential land suitability can be grouped to:

a. Plantation crops (coconut, cocoa, clove, and robusta coffee) range from unsuitable (N), quite suitable (S2), marginally appropriate (S3). Land quality as a limiting factor is: quality of land erosion hazard (slope and erosion hazard), temperature, water availability (rainfall and dry moon), and rooting medium (soil texture and crude material).

b. The horticultural crops of fruits (bananas, oranges, durian, jackfruit, rambutan, mangosteen, and mango) range from unsuitable $(\mathrm{N})$, quite suitable (S2) marginally suitable (S3), and highly sitable (S1). Land quality that is an inhibiting factor for the development of fruits horticultural crops are: quality of land erosion hazard (slope and erosion hazard), quality of water availability (rainfall, dry moon), temperature, root medium (soil texture and crude material)

Based on the assumption of the level of improvement and the type of improvement effort on the actual quality/characteristics of the land to be potential according to the level of management, the suitability of potential land for evaluated commodities (plantation crops and horticulture fruits) can generally be raised one to two levels.

Heavy to very severe erosion hazard limits in terrain conditions are not too steep can be overcome by conservation actions either by mechanical or vegetative means by planting plant species that can withstand erosion and core reinforcement plants. However, on steep slopes to very steep terrain with very severe erosion hazards, it is very difficult to repair so that potentially remains a limitation in the development of the land concerned. 
Limitations of water availability that are not too steep can still be overcome (rainfall) that is too high in the upper with the addition of organic material. Map region, still remains a limiting factor in the of Potential Land Suitability in Unda development of several commodities of Watershed is presented of Figure 1. plantation crops and horticultural crops of fruits. Similarly, on the barrier duration of dry months in the lower region. Low temperature land quality in some land units

Land Management Directions/ Recommendations

As it is known that land suitability in the upper region is potentially still a evaluation not only produces land limiting factor in the development of some suitability classes, it should also be able to plantation commodities and horticulture of demonstrate further land management fruits.

The quality of rooted soil media (coarse soil texture and high coarse material) is still a constraint for several types of plantation commodities and options. The assumption used is that the land suitability class as described above is grouped based on the type and number of limiting factors.

Establishment of alternative land horticulture of fruits. The rather coarse soil texture with high sand content still remains a barrier for some agricultural commodities because the grasp of soil to water is very low. The high content of crude materials in some units of land is still a limiting factor because it can interfere with the possible improvements to its inhibiting development of plant roots. Rough ground factors (land productivity test) . However, texture with high sand content on slopes this research did not reach the land 
productivity test due to time and budget with the essence of land suitability is constraints. sustainability, terms of use, social culture

The need to design alternative land and economy.

use in the research area is for improvement (sustainability of the land), in addition to increased productivity of land /plants. In accordance with the results of field identification, that dry areas in the research area are mostly very vulnerable to erosion and landslide hazards so that in their mechanical methods or by vegetative management all their rules must be fully methods. In land-use planning, land with understood and adhered to, in accordance slope.
The high level of erosion hazard, vulnerability to landslide, and lack of conservation measures in the research area require first priority on erosion and landslide prevention actions through soil and water conservation measures either by 


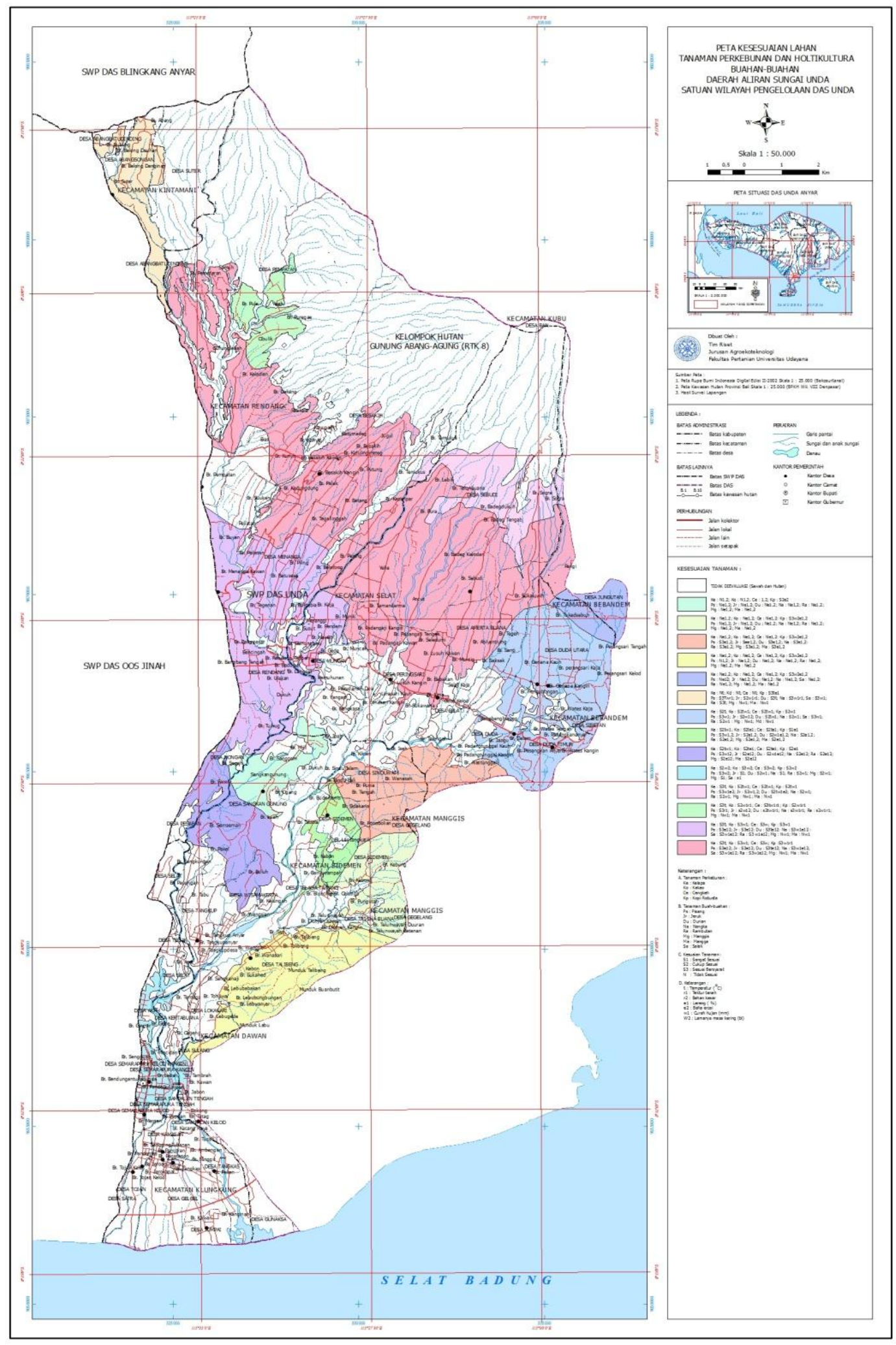

Fig. 1. Map of Potential Land Suitability in Unda Watershed $40 \%$ is not suitable for agriculture and should be left closed to forests (Hardjowigeno and Widiatmaka, 2007) 
As is well known, dryland land in the research area (outside forest areas) is a community-owned land area and is largely a mixed plantation. Therefore, to recommend the community's land to be covered by forest does not seem possible. In FAO (1983) it is explained that the targets that must be achieved by village land use planning must meet the requirements of sustainability, efficiency, equity and acceptability (sustainability, efficiency, equity and acceptability).

As is well known, dryland land in the research area (outside forest areas) is a community-owned land area and is largely a mixed plantation. Therefore, to recommend the community's land to be covered by forest does not seem possible. In FAO (1983) it is explained that the targets that must be achieved by village land use planning must meet the requirements of sustainability, efficiency, equity and acceptability (sustainability, efficiency, equity and acceptability).
Sustainable land management / management with emphasis on sustainability and optimization can be recommended with agroforestry pattern accompanied by erosion prevention and landslide vegetatively because it is cheaper and easy to be done by farmers. In addition, forest crops have high economic value. However, it should be noted that in the implementation of vegetative methods several things to be considered are: selection of plants, (slope level), and their vulnerability to erosion and landslides.

As a vegetative requirement for landslide control, plant species that are eligible have the characteristics of deep roots (reaching rocks), firm roots and binding of soil aggregates and the biomass weight is light. So the suggested cultivation effort is a combination of forest timber plantation with horticultural crop of fruits with minimum requirement of $40 \%$ must be covered with forest wood including erosion prevention and landslide plant by paying attention to plant density. The types of 
wood forest that can be developed is the that can be recommended according to local types of wood that canopy is not too heavy, biophysical conditions are: Aleurites growing fast like gempinis, jabon, and moluccana (Kemiri), Vitek pubescens types of flowers such as butterflies Albisia. (Laban), Lagerstroemia speciosa (Bungur), Particularly on lands with steep Melia azedarach (Mindi), Cassia siamea inclination rates (45-65\%) to very steep (> (Johar), Acacia villosa, Eucalyptus alba, $65 \%$ ) and landslide prone, plant density Leucaena glauca. Especially on the cliffs of should be distinguished between the slope, the cliff and right left river can be the central slope and the upper/peak slopes. recommended bamboo plants.

At the foot of the closing slope closest to the standard of plant density, the middle of

\section{CONCLUSIONS}

the slope closure is somewhat rare ie $1 / 2$ density standard, and the top of the rare closure is $1 / 4$ standard of plant density. At rare densities filled with cover crops (cover crop). In the center and upper part of the slope, good drainage (internal and external) improvements are made so that the water are:

entering the soil is not too large so that the water saturation level on the soil above the impermeable layer (stripping plane) can be reduced.

Several types of erosion and landslide prevention plants which have deep root roots and numerous root roots of the land that are the main obstacle factors

The actual land suitability for plantation crops and horticultural crops of fruits in the study area is largely classified as conditional (S3) to nonconforming(N).The quality/characteristics

1. Quality of land erosion hazard, steep slopes, availability of water (rainfall and dry moon), annual average temperature / temperature, root medium (soil texture and crude material).

2. Land suitability for plantation crops 
(coconut, cocoa, clove, and robusta

coffee) ranges from unsuitable $(\mathrm{N})$, quite appropriate (S2), marginally suitable (S3). Land quality that is a limiting factor is: quality of land erosion hazard (slope and erosion hazard), temperature, water availability (rainfall and dry moon), and root medium (coarse-coarse soil texture).

3. Land suitability for horticultural crops of fruits (bananas, oranges, durian, jackfruit, rambutan, mangosteen, and mango) ranges from unsuitable (N), quite appropriate (S2), marginal suitable (S3), and highly suitable (S1). Land quality that is an inhibiting factor for the development of fruits horticultural crops are: quality of land erosion hazard (slope and erosion hazard), quality of water availability (rainfall, dry moon), temperature, root medium (soil texture and crude material)
4. Sustainable land management/ management with emphasis on sustainability and optimization of land is recommended with agroforestry pattern which is accompanied by erosion prevention and landslide vegetatively because it is cheaper and easy to be done by farmers

5. Some types of erosion prevention and landscaping plants which can be recommended according to local biophysical conditions are: Aleurites moluccana (Kemiri), Vitek pubescens (Laban), Lagerstroemia speciosa (Bungur), Melia azedarach (Mindi), Cassia siamea (Johar) Acacia villosa, Eucalyptus alba, Leucaena glauca. Especially on the cliffs of the cliff and right left river can be recommended bamboo plants.

\section{REFERENCES}

Asdak, C. (2007). Hidrologi dan Pengelolaan Daerah Aliran 
Sungai. Yogyaarta : Gadjah Puslittanak. (1993). Petunjuk Teknis Mada Press..

Arsyad, S. (2010). Konservasi Tanah dan Air. Bogor: IPB Press.

Balai Pengelolaan DAS Unda Anyar. (2010). Laporan Karakteristik DAS Unda. Balai Pengelolaan Daerah Aliran Sungai Unda Anyar. Direktorat Jenderal Rehabilitasi Lahan dan Perhutanan Sosial. Departemen Kehutanan.

Departemen Kehutanan. (2009). Peraturan

Menteri Kehutanan Republik Indonesia Nomor: P. 39/MenhutII/2009 Tentang Pedoman Penyusunan Rencana Pengelolaan Daerah Aliran Sungai Terpadu.

Djaenudin, D., Marwan, H., Subagjo, H., \& A. Hidayat. (2003). Evaluasi lahan untuk komoditas pertanian. Balai Penelitian Tanah. Pusat Penelitian dan Pengembangan tanah dan Agroklimat Bdan Litbang Pertanian Departemen Pertanian.

LREPP II. (1994). Kesesuaian lahan untuk tanaman pertanian dan tanaman Kehutanan. Laporan Teknis No. 7. Versi 1.0 April 1994.

PPT. (1983). Lampiran Term of Reference Klasifikasi Kesesuaian Lahan. Pusat Penelitian Tanah. Kerjasama dengan Proyek Penelitian Pertanian Menunjang Transmigrasi (P3MT). Badan Penelitian dan Pengembangan Pertanian Departemen Pertanian

Puslittanak. (1993). Peta Tanah Semi Detil Skala 1 : 50.000. Pusat Penelitian Tanah dan Agroklimat. Kerjasama dengan Proyek Pembangunan Penelitian Pertanian Nasional Badan Penelitian dan Pengembangan Petanian Departemen Pertanian. Evaluasi Lahan. Pusat Penelitian Tanah dan Agroklimat. Kerjasama dengan Proyek Pembangunan Penelitian Pertanian Nasional Badan Penelitian dan Pengembangan Petanian Departemen Pertanian.

Azhari, S. (1997). Etika lingkungan dalam pembangunan berkelanjutan. Direktorat Jenderal Pendidikan Tinggi Departemen Pendidikan dan Kebudayaan.

Hardjowigeno, S., \& Widiatmaka. (2007). Evaluasi Kesesuaian Lahan dan Perencanaan Tataguna Lahan. Yogyakarta: Gadjah Mada University Press.

Trigunasih, N. M., Lanya, I., Subadiyasa, I. N. N., \& Adnyana, I. M. (2016). Mapping Land Suitability of Subak on base on Geographyc Information System (GIS) in Denpasar, Bali. Internatioanl Journal of Bioscience and Biotechnology, 4 (1): 16-28. 